\title{
Milk stage kernel infection frequency in kharif sorghum at grain mold prone locations in India
}

\author{
I.K. DAS ${ }^{*}$, C. GOVARDHAN² , R.B. GHORADE ${ }^{3}$, Y.D. NARAYANA ${ }^{4}$, G.R. BHANDERI ${ }^{5}$, N. KANNABABU ${ }^{1}$, SUNIL \\ KUMAR $^{1}$ and V.A. TONAPI ${ }^{1}$ \\ ${ }^{1}$ Indian Institute of Millets Research, Hyderabad 500 030,Telangana, India \\ ${ }^{2}$ Department of Genetics, Osmania University, Hyderabad 500 007, Telangana, India \\ ${ }^{3}$ Panjabrao Deshmukh Krishi Vidyapeeth, Akola 444 104, Maharashtra, India \\ ${ }^{4}$ University of Agricultural Sciences, Dharwad 580005 , Karnataka, India \\ ${ }^{5}$ Navsari Agricultural University, Surat 395 007, Gujarat, India
}

Received: 21 March 2017/ Accepted: 17 May 2017/ Published online: 25 May 2017

CIndian Phytopathological Society 2017

\begin{abstract}
Milk stage kernel infection frequency has substantial effects on mold development in mature sorghum grain. Field experiments were conducted with 16 sorghum genotypes at four grain mold prone locations (Akola, Hyderabad, Dharwad and Surat) in India during kharif 2014 and 2015. The genotypes included grain mold susceptible, resistant, improved germplasm, breeding lines and released cultivars. The objective was to quantify natural infection frequency of fungi in milk stage kernel and study the response of various sorghum genotypes against this infection. Results revealed that Fusarium (16.1\%) and Curvularia spp. (7.9\%) were predominant fungal genera at the milk stage kernel at all the locations under study. Few other fungi namely Alternaria, Bipolaris, Aspergillus, and Penicillium spp. were detected sporadically in low frequency $(\sim 0.95 \%)$. There was significant negative association between infection frequency of Fusarium and Curvularia especially when intensity of infection was moderate to high (>25\%). The genotypes B58586, GMR156-1, GMR166-1, SGMRN12-3-1 and IS25070 were found promising with low intensity (average total infection $<10 \%$ ) of infection across environments. The information can be useful for resistant breeding program against specific fungal component of grain mold.
\end{abstract}

Keywords: Curvularia, Fusarium, interactions, milk stage infection frequency, Sorghum

Sorghum is one of the most important crops in semiarid tropics covering a harvested area of around 6.3 million ha in India (Das and Padmaja, 2016). Grain mold is a serious disease of sorghum especially when the crop is grown during the rainy season (Thakur et al., 2006). The disease affects grain yield, seed and grain quality, marketability of grain and occasionally contaminates grain with harmful mycotoxins (Das et al., 2012b). Many fungi including parasites and saprophytes are responsible for causing grain mold in sorghum. Grain mold pathogens or parasites are capable of infecting sorghum florets at the time of anthesis. Initial infection and subsequent colonization pattern of sorghum spikelet tissues by grain mold fungi are established (Forbes et al., 1992). However, there was major focus on identification of resistance to 'grain weathering' that involves colonization of grain by saprophytes during the post-maturity stage. Recent studies revealed that milk stage (MS) kernel infection frequency is an important parameter that gives an indication of grain mold susceptibility in kharif sorghum (Das et al., 2014). Immature seed, as young as 8 days after anthesis (viz., milk stage), could develop sign of fungal infection (Fig. 1). Fusarium and Curvularia were the major fungal pathogens during this stage followed

${ }^{*}$ Corresponding author: das@ millets.res.in
Alternaria and Bipolaris spp. on sorghum cultivars grown at the research farm of IIMR (Indian Institute of Millets Research), Hyderabad (Das et al., 2014). Such information is important for development of grain mold resistant lines against specific fungi. Identification of host resistance against pathogenic fungi by focusing on early infection stages would be useful. As the number of pathogenic fungi is a few (compared to large number of fungi observed on mature molded grain) and their

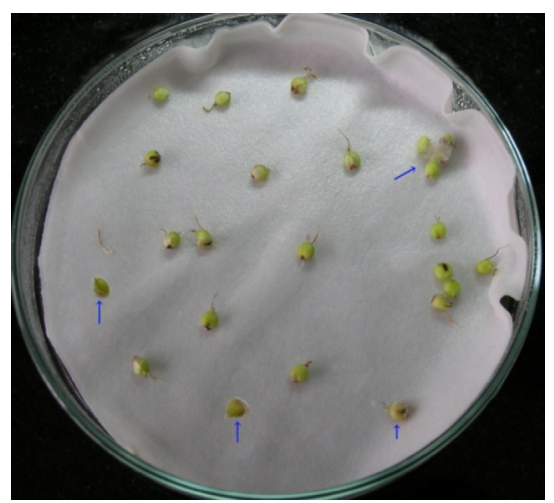

Fig. 1. Fungal colonization on 10-day-old milk stage kernel of sorghum (arrow) when incubated at $28 \pm 1^{\circ} \mathrm{C}$ with $12 \mathrm{~h}$ alternate cycles of light and dark for 7 days 
infection occurs in physiologically active floral tissues there is greater chance of identification and management of resistance. Thakur et al. (2003) studied grain mold variability in fungal complex on mature sorghum grain. At present the milk stage infection frequency of grain mold pathogens are available only for Hyderabad location and lacking for other grain mold prone areas in India. Present study aims to quantify natural infection by grain mold pathogens of sorghum at the milk stage at major grain mold prone areas in India.

\section{MATERIALS AND METHODS}

\section{Sorghum genotypes}

A set of 16 sorghum genotypes were used for fungal diversity studies across four grain mold prone locations in India (Akola, Hyderabad, Dharwad and Surat). The genotypes included one grain mold susceptible (BulkY), one resistant (B58586), one color grain line (IS25070), five improved recombinant inbred lines (GMR124-1, GMR156-1, GMR166-1, GMR83-1, GMR84-2), six improved breeding lines (AKMGR 101, AKMGR 104, PSVGS106, R 10-MP 13, SGMRN 12-3-1, SU 1363) and two released cultivars (CSH23, CSV17) (Table 1).

\section{Field experiments}

Field experiments were conducted at six environments (4 locations during 2014 and 2 locations during 2015). The locations included the sorghum research farms at Panjabrao Deshmukh Krishi Vidyapeeth, Akola; University of Agricultural Sciences, Dharwad; Navsari Agricultural University, Surat and IIMR, Hyderabad during rainy seasons of 2014 and 2015 . The trial was laid out in randomized block design with two replications in a grain mold screening block with single row of $4 \mathrm{~m}$ length, with $60 \mathrm{~cm}$ row spacing. Standard crop management practices were followed to raise the crop. Eight uniform flowering plants in each row were labeled for further observations. The experimental sites received good rainfall and had high relative humidity in both the years (data not shown).

\section{Seed sampling and assay for fungal infection}

Sorghum seeds were sampled from labeled panicles at milk stage (around 10 days after flowering). Milk stage seed was selected for fungal isolation because this was the earliest possible stage when seed could be sampled without much damage. Panicle branches containing seed were randomly sampled from all parts of panicle (base, middle and top). Seeds were carefully separated from panicle branches without causing damage. Seed samples from eight labeled panicles in a cultivar were pooled to form one replicate. Samples were used for isolation of fungi in a laboratory. Assay for seed-borne fungal infection was done as described by Das et al. (2012a). One hundred seeds from each replication were used to study seed-borne fungi. Twenty-five surface sterilized ( $4 \%$ $\mathrm{NaOCl}$ ) seeds were placed on sterile, wet filter paper in a Petri dish humidity chamber prepared by lining the lower lid with a thin layer of absorbent cotton followed by two layers of blotter paper. Seeds were incubated at $28 \pm$ $1^{\circ} \mathrm{C}$ in a BOD incubator with $12 \mathrm{hr}$ alternate cycles of light and dark for 7 days. Sterile water was added in Petri dishes when required to keep the paper moisten for promoting fungal growth. After incubation, samples were observed for number of seeds infected with fungi using a magnifying glass. Seed showing even slight growth of fungus was counted as positive for fungal infection. Infection percentage was calculated on 100 grains from 4 Petri dishes.

\section{Fungal identification}

Identification of seed-borne fungi was done by microscopic observations following procedures described in fungus identification manuals (Leslie and Summerell,

Table 1. Details of sorghum genotype used in the study

\begin{tabular}{lll}
\hline Genotype & Contributing center & Characteristics \\
\hline AKMGR101 & PDKV, Akola & Improved breeding line (STR 293 x 89058-25) \\
AKMGR104 & PDKV, Akola & Improved breeding line (STR 293 x 426-2-64) \\
CSH23 & IIMR, Hyderabad & Released hybrid (7B x RS627) \\
CSV17 & MPUAT, Udaipur & Released variety (SPV946 x SPV772) \\
GMR124-1 & IIMR, Hyderabad & Grain mold tolerant selection from RIL (296B x B58586) \\
GMR156-1 & IIMR, Hyderabad & Grain mold tolerant selection from RIL (296B x B58586) \\
GMR166-1 & IIMR, Hyderabad & Grain mold tolerant selection from RIL (296B x B58586) \\
GMR83-1 & IIMR, Hyderabad & Grain mold tolerant selection from RIL (296B x B58586) \\
GMR84-2 & IIMR, Hyderabad & Grain mold tolerant selection from RIL (296B x B58586) \\
IS25070 & IIMR, Hyderabad & Red grain sorghum germplasm \\
PSGVS106 & RARS, Palem & Improved breeding line \\
R10-MP13 & IIMR, Hyderabad & Improved breeding line \\
SGMRN12-3-1 & UAS, Dharwad & Improved breeding line \\
SU1363 & MPUAT, Udaipur & Improved breeding line \\
BulkY & IIMR, Hyderabad & Grain mold susceptible genetic stock \\
B58586 & IIMR, Hyderabad & Grain mold resistant genetic stock \\
\hline
\end{tabular}


2006). Characteristics of fungal colonies on seed surface were recorded using a magnifying glass and spore characters using a compound microscope (Olympus, BX50). Sample was observed under compound microscope fitted with 10x, 20x, 40x and 100x objectives and a $10 x$ eyepiece. Percentage of seed infection was calculated for each fungus and cultivar.

\section{Data analysis}

Data were analyzed using statistical software (Statistix, version 8.1). Variance for individual year was analyzed separately to test the significance of differences among different parameters. The error variances in the field experiment conducted in two years were heterogeneous, as revealed by Bartlett's test. Therefore, two years data were not combined for analysis. ANOVA for the percent seed infection by different fungi over different locations was performed year wise. Arcsine transformations of original data for percentage of seed infection by Fusarium and Curvularia were carried out. Simple linear regression analysis was performed using Fusarium infection on MS seed as independent and Curvularia infection as dependent variables to study the interaction between these two major pathogens for floret infection. Analysis of variance approach was adopted to test the significance of linear regression. Regression charts were prepared using Microsoft Excel (MS Office 2007).

\section{RESULTS AND DISCUSSION}

\section{Fungal genera identification}

Fusarium spp. appeared as pinkish white to light orange powdery colony on seed with abundant microconidia. Microconidia were in short or long chain, or in false head on conidiophores. Curvularia spp. produced shiny black colonies on seed surface. Conidiophores arose singly or in group bearing 3-septated conidia, which were clavate and almost always slightly curved. Alternaria spp. produced grayish black, often sparse colony on seed surface. Conidiophores produce darkly pigmented conidia. Bipolaris spp. produced dark black colony, conidiophores usually arose singly or in groups and bore conidia in an acropleurogeneous fashion. Aspergillus spp. produced masses of yellow-green ball like structure on a long stalk, conidiophores erect, simple unbranched, colorless, and conidia produced in chains. Penicillum spp. were characterized by gray or olivaceous green colony, conidiophores branched, resemble a broom, conidia round, thick walled produced in chains.

\section{Fungal genera and frequency at different locations}

Frequency of fungal infection in milk stage kernel of sorghum at different grain mold prone locations was highly variable (Fig. 2). Six different fungal genera were recorded across locations. They belonged to Fusarium, Curvularia, Alternaria, Bipolaris, Aspergillus and Penicillium spp. Of these, Fusarium spp. was the most dominant at all the locations except Akola where Curvularia spp., was the most frequent (Fig. 2). Fusarium infection was 1 to 9 times more than Curvularia depending on locations. Infection frequency of Fusarium app. varied from 6.0 to $29.5 \%$, Curvularia spp., 2.1 to $20.7 \%$ and other fungi (other than Fusarium and Curvularia spp.) 0.5 to $6.2 \%$ across locations. Average infection frequencies of the other fungi over six environments (4 locations in 2014 and 2 locations in 2015 ) were $\sim 0.95 \%$ and ranges were 0 to $1.7 \%, 0$ to $1.0 \%, 0.2$ to $2.0 \%$ and 0 to $1.8 \%$, respectively, for Alternaria, Bipolaris, Aspergillus, and Penicillium spp. Pathogenecity of Fusarium and Curvularia spp. were confirmed by inoculation on sorghum florets. Fusarium and Curvularia spp. are major fungi responsible for grain mold in mature sorghum (Govardhan and Das, 2015). This report confirmed that they can infect floret at the time of anthesis and they are pathogens of grain molds. For 'other fungi' it needs to be investigated whether they are true pathogens or just contaminants as they were isolated sporadically in low frequency. All these genera were also reported from Hyderabad locations (Das et al., 2014) and now observed in other grain mold prone locations in India, suggesting their close association with sorghum grain mold. Predominance of Fusarium spp. at Hyderabad and Curvularia spp. at Akola might be due to

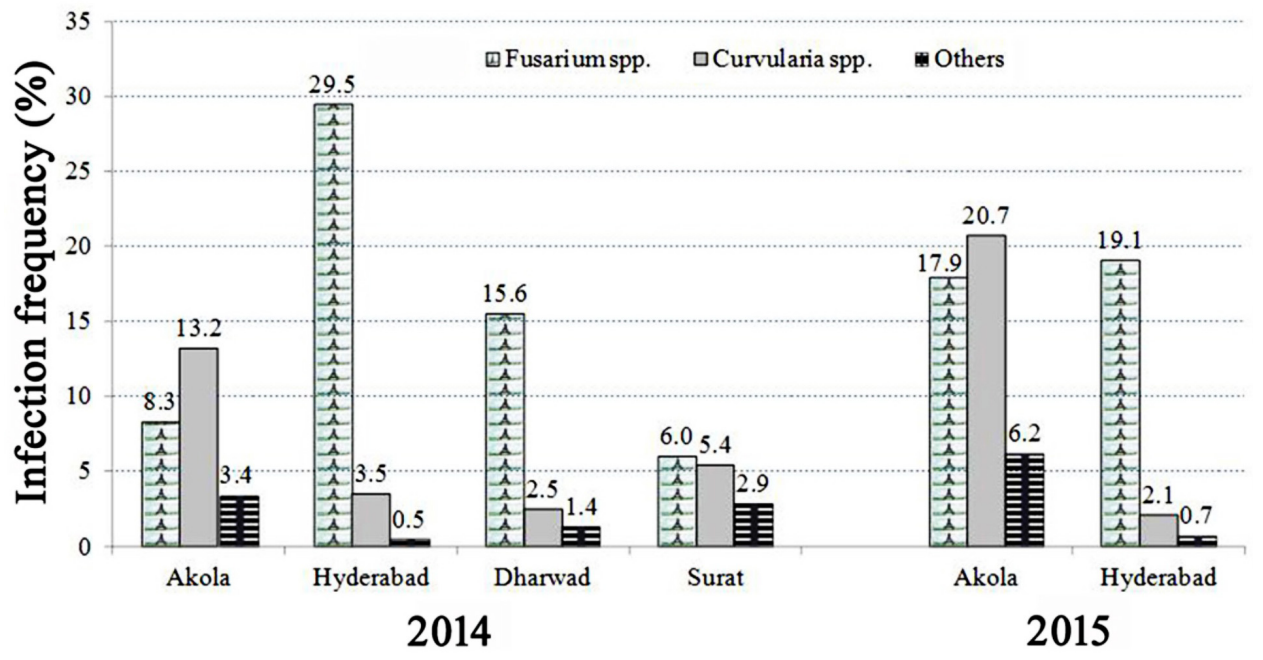

Fig. 2. Natural fungal infection frequencies (\%) on milk stage sorghum kernel at different locations in India during kharif 2014 and 2015 
location-wise variations in fungal population. While studying with mature sorghum grain Thakur et al. (2003) observed significantly high level of Fusarium infection at Parbhani than other locations.

There was variation in total amount of fungal infection over environments (locations and years). During 2014 , frequency of infection was the highest at Hyderabad (33.5\%) followed by Akola (24.9\%), Dharwad $(19.4 \%)$ and the lowest at Surat (14.3\%). During 2015, the highest infection was recorded at Akola (44.8\%) followed by Hyderabad (21.9\%). Mean infections of different fungi were 14.8, 6.2 and $2.0 \%$ for Fusarium, Curvularia and 'other fungi' during 2014, which were 18.5, 11.4 and $3.5 \%$ respectively during 2015 . Year-wise variation might be due to variations in weather parameters (rainfall and relative humidity), which highly influence fungal infection and grain mold development (Indira and Muthusubramanian, 2004).

\section{Interaction between Fusarium and Curvularia infection}

Total infection frequency of the two major fungi (Fusarium plus Curvularia spp.) varied from 2 to $80 \%$ across environments. One of the objectives of this study was to analyse whether infection frequency of Fusarium had any effect on that of Curvularia under natural field conditions and vice-a-versa. The results of regression analysis (Fig. 3) showed that it was the quantum of total infection frequency of these two fungi that determined whether one would have effect or not on the infection frequency of the other. Under low intensity of total infection $(<25 \%)$ there was no significant association between the frequency of infection by Fusarium and Curvularia and vice-a-versa (Fig. 3a) $(p=0.900)$. Under moderate (26 to $50 \%$ ) and high ( $>50 \%$ ) intensity of total infection, however, frequency of Fusarium infection had highly significant negative effects on the frequency of infection by Curvularia and vice-a-versa (Fig. 3b,c) $(p<0.000)$. Overall (0 to $80 \%$ infection) there was significant negative association between these two fungi under natural field conditions (Fig. 3d) $(p=0.045)$. Negligible association at lower infection frequency and high association at higher infection frequency suggested that the fungi required fresh (uninfected) floret for infection. The fact that we did not observed the cases of multiple infection at this stage of the grain, further supported the above view. Early colonization of grain mold fungi was found to be very heavy with a denser growth around the ovary base, leaving less chance for new infection.

\section{Genotypic variation towards milk stage kernel infection}

There was wide variation among the genotypes for fungal infection at milk stage (Table 2). This variation was observed in all the locations and the years. Fusarium infection was the lowest in the resistant B58586 (mean $6.4 \%$, range 0.8 to $13.2 \%$ ) and the highest in the susceptible Bulk $Y$ (mean 29.8\%, range 12.5 to $56 \%$ ) across environments. Among the genotypes GMR156-
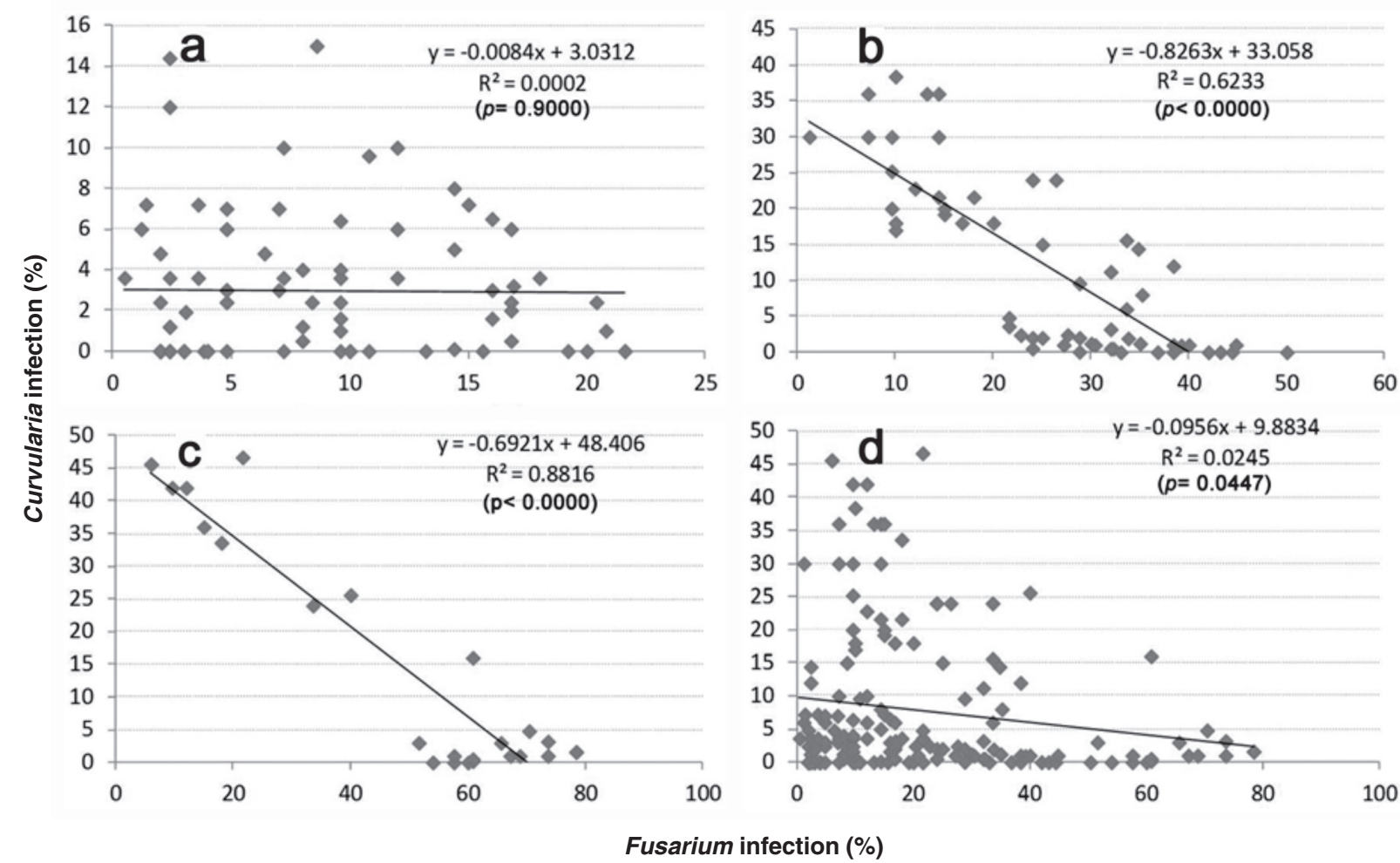

Fig. 3. Interrelationship between Fusarium and Curvularia infection on milk stage kernels of sorghum at different intensity of natural infections. Total infection frequency (Fusarium + Curvularia) (a) less than $25 \%$, (b) 26 to $50 \%$, (c) more than $50 \%$, and (d) overall (0 to $80 \%)$ 
Table 2. Reactions of sorghum genotypes towards milk stage kernel infection at different locations in India during kharif 2014 and 2015

\begin{tabular}{|c|c|c|c|c|c|c|c|c|c|c|c|c|}
\hline \multirow[t]{4}{*}{ Genotype } & \multicolumn{8}{|c|}{2014} & \multicolumn{4}{|c|}{2015} \\
\hline & \multicolumn{2}{|c|}{ Akola } & \multicolumn{2}{|c|}{ Hyderabad } & \multicolumn{2}{|c|}{ Surat } & \multicolumn{2}{|c|}{ Dharwad } & \multicolumn{2}{|c|}{ Akola } & \multicolumn{2}{|c|}{ Hyderabad } \\
\hline & Fus & Cur & Fus & $\overline{C u r}$ & Fus & Cur & Fus & Cur & Fus & Cur & Fus & Cur \\
\hline & spp. & spp. & spp. & spp. & spp. & spp. & spp. & spp. & spp. & spp. & spp. & spp. \\
\hline AKMGR101 & $14(22)^{*}$ & $5(13)$ & 26 & $0(0)$ & $35(36)$ & $1(6)$ & 6 & $0(0)$ & 7 & 35 & 7 & $1(6)$ \\
\hline AKMGR104 & 12(20) & $24(29)$ & 6 & $4(11)$ & $5(12)$ & $25(30)$ & 19 & $0(0)$ & 52 & 0 & 3 & $2(7)$ \\
\hline $\mathrm{CSH} 23$ & $9(17)$ & $7(14)$ & 30 & $1(6)$ & $5(13)$ & $8(16)$ & 23 & $1(6)$ & 10 & 41 & 14 & $4(11)$ \\
\hline CSV17 & $6(14)$ & $34(36)$ & 34 & $5(12)$ & $2(8)$ & $0(0)$ & 30 & $0(0)$ & 4 & 0 & 16 & $5(13)$ \\
\hline GMR124-1 & $13(21)$ & $23(27)$ & 36 & $1(5)$ & 2(8) & $0(0)$ & 16 & $1(5)$ & 8 & 33 & 49 & $0(0)$ \\
\hline GMR156-1 & $1(5)$ & $2(9)$ & 31 & $2(7)$ & $2(7)$ & $0(0)$ & 19 & $1(6)$ & 8 & 1 & 25 & $2(7)$ \\
\hline GMR166-1 & $2(8)$ & $4(11)$ & 13 & $4(12)$ & $1(6)$ & $0(0)$ & 31 & $4(12)$ & 18 & 19 & 4 & $0(0)$ \\
\hline GMR83-1 & $20(26)$ & $18(25)$ & 24 & $4(11)$ & 2(8) & $0(0)$ & 15 & $4(11)$ & 21 & 29 & 4 & $3(10)$ \\
\hline GMR84-2 & $4(11)$ & $7(15)$ & 34 & $3(10)$ & 2(8) & $0(0)$ & 13 & $3(10)$ & 12 & 31 & 45 & $2(8)$ \\
\hline IS25070 & $8(16)$ & $4(11)$ & 44 & $3(10)$ & $1(5)$ & $0(0)$ & 4 & $3(10)$ & 15 & 0 & 36 & $1(7)$ \\
\hline PSVGS106 & $7(15)$ & $23(28)$ & 51 & $4(11)$ & $4(11)$ & $0(0)$ & 11 & $0(0)$ & 16 & 20 & 35 & $0(0)$ \\
\hline R10-MP13 & $12(18)$ & $2(9)$ & 20 & $2(8)$ & $13(21)$ & $9(17)$ & 6 & 2(8) & 36 & 18 & 4 & $7(15)$ \\
\hline SGMRN12-3-1 & $3(9)$ & $5(12)$ & 36 & $2(8)$ & $4(11)$ & $0(0)$ & 7 & 2(8) & 21 & 23 & 4 & $0(0)$ \\
\hline SU 1363 & $4(12)$ & $20(24)$ & 33 & $2(7)$ & $2(7)$ & $0(0)$ & 5 & $0(0)$ & 34 & 15 & 15 & $3(9)$ \\
\hline Bulk Y & 13(21) & $27(31)$ & 56 & $7(15)$ & $17(24)$ & $44(42)$ & 43 & $5(0)$ & 19 & 23 & 31 & $6(14)$ \\
\hline B58586 & $6(14)$ & $9(17)$ & 8 & $13(21)$ & $1(5)$ & $0(0)$ & 2 & $13(21)$ & 8 & 44 & 13 & $1(5)$ \\
\hline CD at $5 \%$ & (4.5) & (7.3) & 6.2 & $(2.4)$ & (2.8) & (1.4) & 4.8 & $(2.4)$ & 4.3 & 4.1 & 3.4 & (1.5) \\
\hline
\end{tabular}

The values are mean of two replications. Seed-borne infection were estimated by placing 100 surface sterilized seeds $(25$ seeds per plate) on sterile, wet filter paper in a Petri dish and incubated at $28 \pm 1^{\circ} \mathrm{C}$ with $12 \mathrm{~h}$ alternate cycles of light and dark for 7 days; Figures in the parentheses are Arcsine transformed values. Fus - Fusarium, Cur - Curvularia

1, GMR166-1, IS25070, and SU1363 performed at par with the resistant B58586 in milks stage Fusarium infection (11.3 to $17.2 \%)$. Curvularia infection, on the other hand, was the lowest in the GMR156-1 (mean $1.4 \%$, range 0 to $2.4 \%$ ) and the highest in the susceptible Bulk Y (mean 18.7\%, range 5.0 to $44.3 \%$ ). Among the genotypes IS25070, SGMRN12-3-1, R10-MP13, and AKMGR101 performed at par with the GMR156-1 in milks stage Curvularia infection (1.8 to $7.1 \%$ ). Considering total fungal infection the genotype B58586, GMR156-1, IS25070, GMR166-1, and SGMRN12-3-1 were promising with low intensity of milk stage kernel infection (average total infection $<10 \%$ ). There was variation in performance of a genotype under different environments which might be due to variations in weather parameters in different location and years. Grain mold disease is known for its high weather dependence (Audilakshmi et al., 2011).

The present study for the first time enumerated natural infection at milk stage kernel of sorghum genotypes at multiple grain mold prone locations in India. Fusarium and Curvularia spp. were predominant fungal genera at this stage of grain development in all the locations under study. Other fungal genera Alternaria, Bipolaris, Aspergillus, and Penicillium spp. were intercepted sporadically in low frequency. There was interaction between Fusarium and Curvularia for infection of sorghum floret especially at high frequency of total infection. The genotypes B58586, GMR156-1 and IS25070 were promising with low intensity of milk stage kernel infection across environment. As frequency of infection at milk stage play significant role in deciding post-physiological maturity grain mold severity (Das et al., 2014), this information can be useful for resistant breeding program against specific fungal component of grain mold.

\section{ACKNOWLEDGEMENTS}

Authors are thankful to the AICRP Sorghum In-charges of the Akola, Dharwad, Palem, Udaipur, Surat and IIMR Hyderabad for supplying seed materials of selected sorghum genotype of their respective centers.

\section{REFERENCES}

Audilakshmi S, Das IK, Ghorade RB, Mane PN, Kamatar MY, Narayana YD and Seetharama N (2011). Genetic improvement of sorghum for grain mould resistance: I. Performance of sorghum recombinant inbred lines for grain mould reactions across environments. Crop Prot. 30: 753758.

Das IK, Audilakshmi S, Annapurna A, Kannababu N and Patil JV (2012a). Relationship among seed germination and other characters associated with Fusarium grain mold disease in sorghum (Sorghum bicolor L. Moench) using path coefficient analysis. Can. J. Pl. Path. 34: 203-212.

Das IK, Audilakshmi S and Patil JV (2012b). Fusarium Grain Mold: The major component of grain mold in sorghum. The Eur. J. Pl. Sci. Biotechnol. 6: 45-55.

Das IK, Govardhan and Patil JV (2014). Milk stage kernel infection frequency and its relationship with grain mold development in sorghum. Indian J. Plant Prot. 42: 389-397. 
Das IK and Padmaja PG (2016). Biotic stress resistance in sorghum. Acad. Press, USA, 246p.

Forbes GA, Bandyopadhyay $R$ and Garcia G (1992). A review of sorghum grain mold. Sorghum and millets diseases: A second world review. de Milliano WAJ, Frederiksen RA, Bengston GD (Eds.), International Crops Research Institute for the Semi-Arid Tropics (CP738), Patancheru. pp. 265-272.

Frederiksen RA (1986). Compendium of Sorghum Diseases. The American Phytopathological Society and Department of Plant Pathology and Microbiology, Texas A\&M University, Texas, USA, $82 \mathrm{pp}$

Govardhan C and DAS IK (2015). Minimization of floret infection by fungi causing grain mold in sorghum (Sorghum bicolor) through use of fungicides. Indian Phytopath. 68: 67-72.
Indira S and Muthusubramanian V (2004). Influence of weather parameters on spore production in major mold pathogens of sorghum in relation to mold severity in the field. Ind. J. Pl. Prot. 32: 75-79.

Leslie JF and Summerell BA (2006). The Fusarium laboratory manual. Blackwell Publishing Asia, 550 Swanston Street, Carlton, Victoria 3053, Australia. 388 pp.

Thakur RP, Rao VP, Navi SS, Garud TB, Agarkar GD and Bharathi Bhat (2003). Sorghum grain mold: Variability in fungal complex. Int. Sorghum \& Millet Newslett. 44: 104108.

Thakur RP, Reddy BVS, Indira S, Rao VP, Navi SS, Yang XB and Ramesh S (2006) Sorghum grain mold. Information Bulletin No. 72. ICRISAT, Patancheru 502 324, Andhra Pradesh, India. 32pp. 